\title{
Factores que influyen en la intención de uso de las tarjetas de crédito por parte de los jóvenes universitarios
}

\author{
Factors that influence college students' intention to use credit cards
}

\author{
Maria Camila Bermeo-Giraldo \\ Ingeniera Industrial, Institución Universitaria Escolme, Medellín- Colombia, \\ cies2@escolme.edu.co \\ Lyssed Álvarez-Agudelo \\ Estudiante de Tecnología en Gestión en Finanzas, Institución Universitaria Escolme, \\ Medellín - Colombia, lysruby@hotmail.com \\ Mónica Inés Ospina-Rúa \\ Estudiante de Tecnología en Gestión en Finanzas, Institución Universitaria Escolme, \\ Medellín - Colombia, miospinar@escolme.edu.co \\ Yesenia Acevedo-Correa \\ Socióloga, Fundación Universitaria Católica del Norte, \\ Medellín -Colombia, yacevedoc@ucn.edu.co \\ Iván Alonso Montoya-Restrepo \\ PhD en Ciencias Económicas, Universidad Nacional de Colombia, \\ Medellín -Colombia, iamontoyar@unal.edu.co
}

Cómo citar / How to cite

Bermeo-Giraldo, M.C., Álvarez-Agudelo, L., Ospina-Rúa, M., Acevedo-Correa, Y., Montoya-Restrepo, I. (2019). Factores que influyen en la intención de uso de las tarjetas de crédito por parte de los jóvenes universitarios. Revista CEA, 5(9), 77-96. https://doi.org/10.22430/24223182.1257

Recibido: 13 de septiembre de 2018

Aceptado: 20 de noviembre de 2018

\section{Resumen}

El artículo identifica los factores asociados al uso de las tarjetas de crédito en los jóvenes universitarios. La investigación se realizó bajo un análisis factorial exploratorio, con un enfoque cuantitativo y de alcance exploratorio-descriptivo. Para el análisis de datos se cuantificaron los niveles de asociación entre los constructos del modelo conceptual en intención de uso de las tarjetas de crédito, mediante el coeficiente $V$ de Cramer. Los resultados verificaron la capacidad explicativa del modelo y encontraron la asociación entre los factores: educación financiera, beneficios percibidos del crédito, intención de uso de tarjetas de crédito, cultura del ahorro y notificación de costos por parte de la entidad bancaria. Se concluye que las relaciones presentes en el modelo demuestran que 
una de las variables más importantes al momento de fomentar y promocionar el uso de las tarjetas de crédito está estrechamente vinculado con la satisfacción percibida por el cliente, incluyendo desde la atención prestada por el asesor corporativo hasta el análisis de la relación entre el conocimiento financiero con el que cuenta el usuario, y la relación costo-beneficio que recibe de la entidad al adquirir el crédito.

Palabras clave: modelo de usabilidad, tarjetas de crédito, educación financiera, estudiantes universitarios.

\begin{abstract}
This study identifies the factors associated with the use of credit cards by college students. It employs exploratory factor analysis with a quantitative approach and an exploratory-descriptive scope. The levels of association between the constructs in the conceptual model of the intention to use credit cards are quantified by means of Cramér's $V$ coefficient. The results confirmed the explanatory power of the model and revealed associations between several factors: financial education, perceived benefits of the credit, intention to use credit cards, savings culture, and fee notification by the banking institution. The relationships in the model demonstrate that one of the most important variables to promote the use of credit cards is closely linked to the satisfaction perceived by the customer; this includes from customer care by the corporate advisor to the analysis of the relationship between the financial knowledge of the user and the cost-benefit relationship received from the banking institution when the credit is obtained.
\end{abstract}

Keywords: usage model, credit card, financial education, university students.

\title{
1. INTRODUCCIÓN
}

En Colombia se ha comprobado que las personas obtienen créditos sin estar seguros de tener la capacidad de cubrir sus deudas, recurriendo al pago de muchos de los productos de la canasta familiar por medio de las tarjetas de crédito (Banco de la República, 2014). De acuerdo con ello, el Banco de la República de Colombia (2014), sugiere a los ciudadanos ser prudentes al solicitar tarjetas de crédito o créditos de consumo, ya que la obtención de estos ha aumentado progresivamente durante los últimos años, dejando como resultado un alto endeudamiento en los colombianos (Gómez, Echeverri, \& Roca, 2016).

Para el año 2016, «la carga financiera de los hogares no sufrió un aumento para este periodo, pero la proporción de deuda que se concentra en los hogares vulnerables, sí aumentó» (Pacheco-Bernal, Segovia-Baquero, \& Yaruro-Jaime, 2017, p. 1). Entre las causas de este endeudamiento se reportó el uso excesivo de las tarjetas de crédito, ya que estas representan uno de los medios más utilizados por las familias en Colombia para satisfacer las necesidades y solucionar problemas de financiación (Banco de la República, 2014).

Otro factor fundamental, que puede ser determinante en el incremento del uso de las tarjetas de crédito es el aumento del desempleo que ha enfrentado el país, lo cual conlleva a los ciudadanos a tener que recurrir a estos préstamos para poder pagar gastos habituales o tener dinero de forma inmediata. Lo anterior, sustentado en que la administración de estas tarjetas, según Goldenberg 
(2017) constituyen un mecanismo de acceso al crédito que trae consigo dificultades técnicas para el portador, en cuanto a la definición de los conceptos relevantes, como su naturaleza jurídica, la difícil comprensión financieras que se tiene desde el momento de la obtención, de la utilización y de elegir la forma de pago.

Por otro lado, Diniz, Rogers, Barboza, \& Mendes-Da-Silva (2016) aconsejan a los portadores de tarjetas de crédito, y a quienes deseen adquirirlas, hacer uso responsable de ellas para convertirlas en aliado del control financiero personal, ya que la tarjeta de crédito es una forma de pago o una herramienta para manejar gastos, pero no es una extensión de los ingresos. En este sentido, utilizar la educación financiera y la cultura del ahorro como una guía que aumenta el conocimiento sobre el uso del crédito, los tiempos de compra y los conceptos financieros, disminuye el endeudamiento (Araújo \& Souza, 2012).

Para el caso de los estudiantes universitarios, tener una economía personal estable es importante porque evitaría afectaciones financieras o una marcación negativa ante las entidades financieras en el historial crediticio, esto de acuerdo con el estudio realizado por Neme-Chávez \& Forero-Medina (2018), hay una simbología que conlleva a que estos sujetos manifiesten temor y rechazo hacia su uso porque llevarían a un consumo desmedido. De allí que, Limbu, Huhmann, \& Xu (2012), mencionen que esta población es más vulnerable que los adultos mayores en utilizar abusivamente las tarjetas de crédito en el consumo. Asimismo, Wang (2011) señala que los estudiantes son quienes presentan un comportamiento irresponsable en el uso de las tarjetas de crédito, el cual esta atribuido a la falta de una adecuada educación financiera y cultura de ahorro. Lo que impacta negativamente las posibilidades de ingresar posteriormente al mercado laboral por consecuencia de las deudas excesivas contraídas. Sumado a esto, la falta de comprensión de los costos asociados al uso de las tarjetas de crédito tiene relevancia en el aumento de la adquisición de este medio de pago, ya que elementos como la educación financiera proporcionan habilidades básicas para calcular las tasas de interés y planificar el tiempo de pago (Moreno, García, \& Gutierrez, 2017). También el desconocimiento o notificación de dichos costos podrían determinar la intención de uso de estas tarjetas de crédito por parte de los jóvenes universitarios, ya que si no se conoce la deuda es posible seguir acumulándola. Para Solano, Vega, \& Cárdenas (2015), la cultura de ahorro es un elemento del que carecen los jóvenes entre los 15 y 29 años, que se ve reflejado en problemas en el uso de las tarjetas de crédito, las cuales han presentado un crecimiento para el consumo de bienes. Ello, aumenta la intención de uso de estas por partes de los jóvenes y las posibilidades de experimentar el sobreendeudamiento (Solano et al., 2015). Asimismo, la percepción que tienen los usuarios acerca de los beneficios en la obtención de los créditos al momento de comprar, como diferir las cuotas de pago, acceder a descuentos y demás ventajas de los productos ofrecidos por las entidades bancarias es otro aspecto que influye en la intención de uso de este medio de pago, ya que actúa como un factor de fidelización en su utilización (González, 2016). Estos son factores que procederán a dar estructura a las hipótesis que se describen al detalle en la metodología y que responderán la problemática en cuestión.

De acuerdo con todo lo anterior, esta investigación plantea resolver las preguntas: ¿Qué factores influyen en la intención de uso de las tarjetas de crédito en los jóvenes universitarios? ¿Es posible formular un modelo que refleje el comportamiento de los jóvenes estudiantes frente a la intención de uso de las tarjetas de crédito? En esa misma línea, se ha evidenciado que los estudiantes universitarios, por temor, prefieren el uso de dinero en efectivo más que utilizar tarjetas de crédito, 
lo cual también se encuentra vinculado a la participación de programas de manejo financiero, lo que influye en una comprensión subjetiva de este (Neme-Chavez \& Forero-Medina, 2018).

Para abordar esta problemática, la presente investigación tiene como objetivo identificar los factores asociados al uso de las tarjetas de crédito en los jóvenes universitarios de Medellín y generar nuevas combinaciones de factores para la enunciación de un modelo que refleje el comportamiento de los jóvenes estudiantes frente a la intención de uso de este medio de pago.

\section{MARCO TEÓRICO}

A continuación, se presenta una revisión bibliográfica para la comprensión de la temática, iniciando con la contextualización del problema, la definición de algunos conceptos como la educación financiera y antecedentes o referentes teóricos para la identificación y determinación de los factores que influyen en el uso de las tarjetas de crédito por parte de los jóvenes universitarios y posteriormente, generar el modelo de hipótesis a validar.

\section{Contextualización de la problemática}

Con el paso del tiempo y la evolución tecnológica, el hombre ha modificado los medios para realizar transacciones. Uno de los mecanismos más fáciles para hacerlas son las tarjetas de crédito, tal como lo detalla González (2013) y definidas como el medio por el cual la entidad que emite el documento le otorga un crédito al receptor, para emplearlo en compras, pagos y adquisición de bienes o servicios. Estas también permiten al usuario realizar avances en efectivo, cuyo registro en el sistema queda bajo la misma entidad financiera emisora.

Las tarjetas de crédito se expiden mediante un contrato comercial entre la entidad financiera y el cliente adquisidor, con un plástico como soporte de representación de crédito, que se podrá presentar en cualquier local comercial en donde el usuario desee realizar su pago o compra (González, 2013). La entidad financiera a cambio cobrará una comisión por cada transacción que sea realizada.

Según el Banco de la República de Colombia, existe un constante uso de las tarjetas débito y crédito, el cual se refleja en un crecimiento continuo a lo largo de la década. En el año 2000, el número promedio anual de tarjetas de crédito fue 1.951.646; mientras que, en el año 2013, el total de tarjetas al cierre del año correspondía a 13.475.458, lo que demostró un crecimiento del $590 \%$ aproximadamente durante estos 13 años (Gómez et al., 2016). Igualmente, Gómez et al., (2016) argumentan que, a pesar del aumento en la adquisición de estas tarjetas, durante aproximadamente veinte años, el uso de algunas se encuentra limitado y con algunos impedimentos para poder utilizarse de manera masiva, esto por sus altos costos y las dificultades en la forma de pago en algunos locales comerciales, principalmente en los pequeños e informales.

Por otra parte, el uso de las tarjetas de crédito es un fenómeno que sobrepasa fronteras, ya que a nivel mundial pueden ser utilizadas bajo los mismos beneficios de un adquisidor cualquiera, especialmente el de brindar agilidad en el servicio, debido a que no es necesario la intervención de un funcionario de la entidad emisora de la tarjeta, para adquirir un avance en efectivo o hacer un 
pago (Del Pino \& Vázquez, 2004). En este sentido, el uso de las tarjetas está supeditada a las ventajas y desventajas que conoce el portador. De acuerdo con Denegri, Martínez, \& Etchebarne (2007), las tarjetas de crédito pueden servir en situaciones de emergencia por el poco tiempo requerido en el trámite: la aprobación del crédito puede llevarse a cabo en 5 días hábiles, sin codeudores, ni prendas en garantía. En razón a eso, es más fácil solicitar una tarjeta de crédito, que un crédito de consumo tradicional.

En cuanto a las desventajas, Denegri et al. (2007) mencionan: la presencia de altas tasas de interés, el pago de cuota de manejo, la errada creencia de tener dinero extra, los posibles errores en el momento de la liquidación del pago y los pagos con tarjeta de crédito que en ocasiones no permiten obtener descuentos en las compras. Asimismo, ciertas operaciones como los avances en efectivos generan un interés más alto, esto es importante ya que las altas tasas de intereses y los cargos anuales en ocasiones superan los beneficios ofrecidos.

De la misma forma, Reina, Zuluaga, \& Rozo (2006) señalan una importante implicación financiera generada por el uso constante de las tarjetas de crédito, como lo es el aumento de la inflación; esto dado que, al usarlas, se aumenta la adquisición de servicios y de consumo. También, se pueden resaltar varios aspectos positivos en algunas operaciones financieras, entre las que se encuentran el crecimiento en los últimos años de las diferentes instituciones financieras; por ejemplo a nivel internacional, en República Dominicana se orienta los recursos hacia sectores de la economía, para la articulación de la tarjeta de crédito, la cual ha contribuido al crecimiento económico, ofreciendo fuentes de menor cuantía y colocando créditos potenciales, que pueden ser usados como créditos de consumo para satisfacer las necesidades de los usuarios adquiriendo bienes (Reina et al., 2006).

Para los estudiantes universitarios, el uso de las tarjetas de crédito se presenta como un medio para satisfacer necesidades que, subjetivamente, deben ser resueltas sin diferenciar si estas hacen referencia a gastos obligatorios o al cumplimiento de deseos. En ese sentido, ese consumo se refleja en el pago de matrículas universitarias, pero también en viajes, pasar tiempo con amistades, lo que genera cierta satisfacción y sensación positiva y justificada del gasto (Neme-Chavez \& Forero-Medina, 2018).

\section{Educación financiera}

Para el desarrollo de este estudio, se comprende a la educación financiera como el medio por el cual un sujeto percibe, apropia, selecciona y emplea las concepciones adecuadas del uso de tarjetas de crédito y para la toma de decisiones económicas y financieras acertadas (Rivera \& Bernal, 2018). De acuerdo con Xiao \& O’Neill (2016), la educación financiera es relevante para los consumidores, ya que les permite obtener conocimiento sobre la decisión de compra, pues su objetivo es fomentar un adecuado comportamiento que mejore el bienestar financiero, debido a que el mal manejo de las tarjetas de crédito es una consecuencia de la falta de formación y enseñanza en este campo, la cual no se implementa apropiadamente desde que el usuario inicia la vida crediticia. Por tal razón, el sistema Colombiano ha implementados dos leyes: La Ley 1450 de 2011, que establece la obligación que tiene el Ministerio de Educación de incorporar la educación financiera en el programa de competencias básicas; y la Ley 1328 de 2009, que incorpora la educación financiera como principio en la prestación de servicios financieros y se impone como obligatorio cumplimiento para las entidades financieras de desarrollar programas y campañas (Cano, Esguerra, García, Rueda, \& 
Velasco, 2014). De manera que se hace evidente y necesaria la implementación de una educación financiera, para que ayude a desarrollar las capacidades financieras de las personas sobre el uso responsable y acertado de los productos financieros como las tarjetas de crédito (Rivero, Rosado, \& Pérez, 2017). En este sentido, la educación financiera es reconocida por la literatura como un factor influencial en la intención de uso de las tarjetas de crédito como medio de pago.

\section{Referentes teóricos y antecedentes}

La teoría económica sobre consumo e ingreso establece la vinculación entre estas variables. Keynes (1943) introdujo dicha concepción: el consumo depende casi exclusivamente del ingreso, y manifiesta que, aunque influyen otras consideraciones, estas no poseen importancia suficiente; en este sentido, el consumo dependería del ingreso real disponible (Morettini, 2002). La teoría del consumo de Keynes señala que el incremento en el consumo ante un incremento en el ingreso (conocido como la propensión marginal a consumir-PMgC) crece, pero menos que proporcionalmente. También se señala que esta propensión disminuirá a medida que el ingreso aumente.

Finalmente, un concepto expresado por Keynes es la propensión media para consumir (PMeC), entendida como la cantidad del ingreso que se dispone al consumo, es decir, que la PMeC es mayor a la PMgC. Posteriormente Kuznets plantea dos supuestos de consumo como consecuencia de su trabajo econométrico con series: una curva de corto plazo y otra de largo plazo, de forma que la propensión media al consumo es decreciente en el corto plazo, pero las curvas no demostraban que la PMgC es menor que la PMeC (Morettini, 2002). Con base en estos resultados, Duesenberry (1948) citado en Palley (2008), propone la teoría de ingreso relativo, la cual indica una tendencia de origen psicológico para mantener el nivel de consumo y bienestar, condicionada por los ingresos (incluyendo el ingreso percibido en el periodo anterior) que recibe un individuo frente a los ingresos de los demás.

La teoría del ingreso permanente de Friedman (1957) sugiere que el consumo y el ahorro, que desde la concepción keynesiana se habían concebido como dependientes del ingreso corriente, dependen del ingreso futuro esperado del agente (su expectativa de ingreso permanente) y del ingreso no esperado o transitorio. Lo anterior, en virtud de plantear una teoría en la que los individuos quieren maximizar el bienestar que podrían tener en toda su vida, sujetos a la restricción de que deben gastar los recursos obtenidos a lo largo de toda la vida (Fernández-Corugedo, 2009). El autor sugiere que incrementos en el ingreso llevan a los agentes a decidir si son aumentos de corto plazo (o estocásticos), o si se trata de ingresos permanentes. Frente a los ingresos transitorios, Friedman (1957) indica que los mismos no generan una influencia mayor en el consumo corriente. Por su parte, los cambios en el ingreso permanente serían los factores explicativos de los cambios en las elecciones de consumo. El autor sugiere también que la propensión marginal al consumo en el corto plazo es menor que la propensión marginal a consumir de largo plazo, pues los individuos no tienen idea cierta de si el incremento del ingreso será permanente o no. Las implicaciones de esta teoría para explicar el consumo son relevantes, en tanto los individuos solo modifican la cantidad de consumo que realizan, cuando piensan que el ingreso permanente se ha modificado. Así, pérdidas repentinas del ingreso (o temporales) no cambiarían el consumo de los agentes, si mantienen su expectativa de ingreso futuro o de largo plazo, tal como lo expresa Duesenberry (1948) citado en Palley (2008).

En la actualidad, se han generado críticas a la teoría de Friedman. Se dice que los agentes, cuando logran recibir algún ingreso no previsto, tienden a gastar inmediatamente alguna proporción de ese 
ingreso inesperado, lo que es contrario a lo afirmado por este autor, quien manifiesta que ingresos inesperados eventualmente serían ahorrados. Ganong \& Noel, (2016) señalan que, cuando un agente pierde su empleo, ello lo conduce a gastar menos. Y no solamente eso, sino que cuando se reduce su capacidad de crédito para consumo, también se reducen los consumos. Así las cosas, los autores muestran que el consumidor actual es más cortoplacista.

En cuanto al comportamiento de los consumidores al momento de usar las tarjetas de crédito, algunos autores se han preocupado por este fenómeno. Limbu (2017), plantea la aplicación de un modelo de habilidades de información-motivación-comportamiento por sus siglas en inglés-IMB, con el cual, examinar los efectos directos e indirectos del conocimiento acerca de la tarjeta de crédito y la motivación social sobre el comportamiento del mal de estas entre estudiantes universitarios de Estados Unidos. Entre los resultados se destaca la relación negativa entre la falta de conocimiento sobre la temática y el comportamiento negativo de su uso. Por otro lado, Singh, Rylander, \& Mims (2018) encontraron que los estudiantes universitarios no están preparados para usar el crédito de manera responsable, ni para realizar pagos de manera oportuna. Como resultado, se evidencian diferentes comportamientos de pago para la tarjeta de crédito al dividir a los estudiantes universitarios en cuatro segmentos de comportamiento basados en características útiles. El uso de las tarjetas de crédito se ha abordado también desde el punto de vista financiero, los autores Moreno, García \& Gutiérrez (2017) midieron el nivel de conocimiento financiero en jóvenes universitarios sobre la tasa de interés, la inflación, el ahorro, el uso de tarjetas de crédito y el presupuesto. Los resultados muestran que en la universidad los estudiantes tienen el conocimiento y la costumbre de desarrollar presupuestos para planificar sus gastos, pero su nivel de educación financiera es muy bajo en comparación con el resto de las variables que se evalúan.

A pesar de conocerse poco sobre los factores reales que pueden influir en la intención de uso de las tarjetas de crédito por parte de los jóvenes universitarios, es preciso revisar la importancia que le da la literatura de algunas dimensiones como la cultura del ahorro. Además de la educación financiera, la cultura de ahorro se considera relevante, ya que autores como Solano, Vega \& Cárdenas (2015) argumentan que el no contar con una planeación en el manejo de los recursos financieros o de cultura de ahorro se traduce en altas probabilidades de caer en el sobreendeudamiento. Por su parte, Duque, González \& Ramírez (2016) señalan que los jóvenes tienen bajos niveles de cultura de ahorro, ocasionándoles problemas en el manejo del dinero personal, lo que podría llegar a impactar la economía a nivel nacional. Adicionalmente, la cultura de ahorro es importante porque posibilita mejores decisiones para un adecuado uso de las herramientas que el sistema financiero ofrece (Duque et al., 2016). También, algunos investigadores reconocen que los beneficios percibidos por los usuarios en los servicios financieros son un factor que influye en su utilización (Mejía \& Quintero, 2017). De ahí que Raccanello, \& Herrera (2014) indiquen que es necesario que se proporcione información clara acerca de los beneficios que tienes los consumidores en los servicios y productos financieros para garantizar una buena experiencia en su uso.

\section{METOdOLOGÍA}

La investigación se realizó bajo un análisis factorial exploratorio, con un enfoque cuantitativo y de alcance exploratorio-descriptivo, con el fin de identificar los factores asociados al uso de las tarjetas de crédito en los jóvenes universitarios de Medellín y enunciar un modelo que refleje el 
comportamiento de estos frente a la intención de uso de este medio de pago. En total, se encuestaron 121 jóvenes universitarios de la ciudad de Medellín como muestra representativa de la población, cuyo método de selección fue a conveniencia (no probabilístico), es decir, se aplicó el instrumento a quienes se tenía accesibilidad y proximidad (Otzen \& Manterola, 2017). La población correspondió a estudiantes de pregrado de carreras afines a las áreas de mercadeo, finanzas, administración y negocios internacionales, mayores de 18 años. Entre los 18 y 25 años se encontraba el 22,31 \%; entre los 26 a 35 años el 55,37 \%; entre el 36 a 47 años, el 21,49 \%; y de 40 años en adelante el $0,83 \%$. El nivel socioeconómico de los participantes era alto (más de la mitad entre los estratos 4 y 5) y el criterio principal a considerar fue que tuvieran o hubieran tenido tarjetas de crédito. El diseño del instrumento contó con 13 preguntas tipo Likert, con cinco opciones de respuesta: muy de acuerdo, de acuerdo, ni en desacuerdo ni de acuerdo, en desacuerdo, muy en desacuerdo, y no sabe o no responde, con escala 1, 2, 3, 4 y 5 respectivamente; la opción no sabe o no responde, no se encuentra incluida en la escala. También incluyó preguntas de opción múltiple con única respuesta y de tipo cerrada. El análisis factorial exploratorio, se realizó con el propósito de obtener una estructura base del modelo teórico a presentar y posteriormente validar con el análisis factorial confirmatorio. El procedimiento realizado supuso analizar cada conjunto de variables de acuerdo con lo siguiente: en primer lugar, se aplicó AFE a todo el grupo de variables utilizando Máxima Verosimilitud (ML) como procedimiento de extracción de los factores y eligiendo VARIMAX como procedimiento de rotación, para ello se utilizó el software estadístico SPSS. En segundo lugar, se plantea un modelo en el que se busca validar seis hipótesis, de acuerdo con los factores obtenidos después de efectuar el AFE. Finalmente se espera corroborar la estructura factorial de cargas estandarizadas obtenidas para cada variable a través de la utilización del Análisis Factorial Confirmatorio.

Seguido de realizar el AFE, el plan de análisis se desarrolló en primer lugar con la validez convergente, que según Calvo-Porral, Martínez-Fernández, \& Juanatey-Boga (2013) se emplea para probar que los constructos que se espera que estén relacionados los estén, implicando evaluar la fiabilidad del modelo, a través de dos niveles: la fiabilidad de los ítems observables y de los constructos.

Luego, con la validez divergente, se probó que esa correlación en la validez convergente, debía «ser mayor que la que exista con respecto a las medidas propuestas para otro constructo distinto» (Martínez-García \& Martínez-Caro, 2009, p. 28). Ello, empleó un análisis de intervalo de confianza en la estimación de la correlación entre cada par de factores y el análisis de Cronbach para la fiabilidad de la consistencia interna del instrumento. Finalmente, con el coeficiente $V$ de Cramer, se realizó la estimación del modelo estructural propuesto recogiendo las hipótesis planteadas y midiendo el grado de asociación entre los factores. En cuanto a las consideraciones éticas, la presente investigación se desarrolló bajo los principios y lineamientos éticos; en este sentido, para la aplicación del instrumento se contempló la firma de un documento de consentimiento informado que comunicaba a los participantes sobre sus derechos y disposición de resultados en lo concerniente al estudio (NoreñaPeña, Moreno, Rojas \& Malpica, 2012).

\section{RESULTADOS}

El análisis factorial exploratorio - AFE - es el nombre genérico con que se designa a un conjunto de métodos estadísticos multivariados de interdependencia, cuyo propósito principal es el de identificar 
una estructura de factores subyacentes a un conjunto amplio de datos (Pérez \& Medrano, 2010) y constituye uno de los métodos estadísticos más utilizados en la investigación en ciencias sociales, puesto que, su contribución al conocimiento científico, consiste en facilitar el análisis de los patrones de interrelación entre las variables, reducir datos, clasificarlos y describirlos (Frías, 2013), procedimientos que son necesarios a la hora de analizar la varianza común a todas las variables y de este modo minimizar el número de variables con cargas altas en un factor, mejorando así la interpretación de factores (Pérez-Gil, Chacón, \& Moreno, 2000).

\section{Rotación de los factores del modelo}

Con la idea de obtener la estructura factorial, se realizó una rotación de ejes a través del método Varimax (método de rotación ortogonal que minimiza el número de variables que tienen saturaciones altas en cada factor) y se obtuvo que las cincuenta y siete variables analizadas se agrupan en cinco componentes o factores, tal y como se señala a continuación.

Factor 1 (Educación financiera): este componente está asociado a las variables relacionadas con la educación financiera; evaluándose aspectos como: la influencia en la toma de decisiones asertivas en todos los ámbitos económicos, la adquisición de conocimiento sobre cómo hacer un presupuesto personal y la posibilidad de tener una visión financiera inteligente y asertiva.

Factor 2 (Beneficios percibidos del crédito): las variables evalúan los beneficios percibidos de los productos crediticios ofrecidos por el sector bancario; puntualmente mide elementos como: la diversidad de franquicias de tarjetas de crédito (MasterCard, Visa, American Express, entre otros), la posibilidad de diferir las compras en cuotas mensuales, la posibilidad de obtener descuentos en tiendas comerciales, acumular millas y tener algún tipo de asistencia médica o seguros contra robos, y finalmente la ventaja de tener un cubrimiento internacional, permitiendo comprar en otras monedas diferentes a los pesos colombianos.

Factor 3 (Intención de uso de tarjetas de crédito): está asociado a las variables relacionadas con la intención de uso de las tarjetas de crédito, específicamente bajo los criterios de: el crecimiento de la economía personal (ingresos para el consumo), la confianza y comodidad al tomador y el fácil acceso que ofrecen las entidades bancarias al momento de ofertar este producto crediticio.

Factor 4 (Cultura del ahorro): asociado a las variables relacionadas con la evaluación de la cultura de ahorro presente en los jóvenes, dichas variables indagan sobre la premisa de si las tarjetas de crédito inducen a las personas a realizar compras innecesarias, entendiendo que brindan la posibilidad de anticipar una compra y tener el dinero seguro para pagarla en lapso prudente.

Factor 5 (Notificación de costos por parte de la entidad bancaria): estas variables corresponden a aquellas que evalúan las ventajas percibidas por las tarjetas frente al acto de notificar los costos asociados a las tarjetas de crédito por parte de la entidad bancaria.

Luego, en la primera fase del análisis, se logró constatar que los datos recolectados en la investigación no parecen mostrar información redundante, ya que, las cargas factoriales de las variables observables en su mayoría fueron superiores a 0,6 (Bagozzi \& Yi, 1988) logrando que el promedio de cargas factoriales para cada constructo pueda obtener un valor superior a 0,7 (Hair, Anderson, 
Tatham, \& Black, 2007). Posterior a este análisis, en la Figura 1 se presenta el modelo de hipótesis a validar (las líneas indican las hipótesis nulas que se validaron en la investigación).

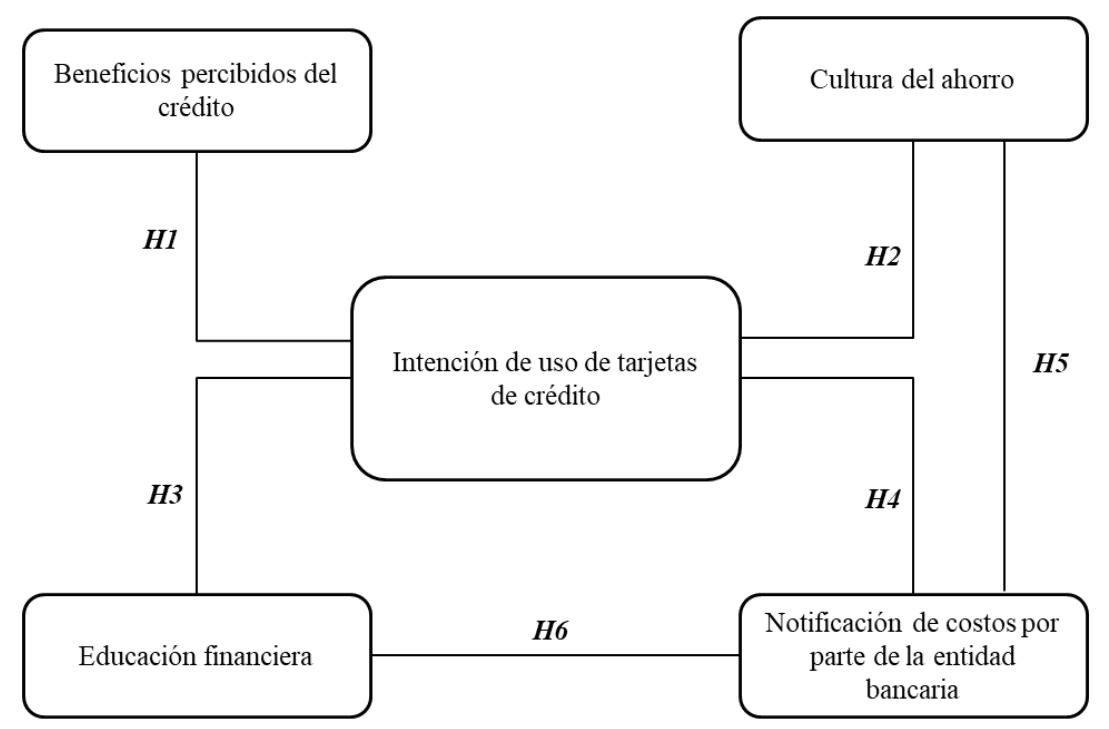

Figura 1. Modelo propuesto para valorar la usabilidad de las tarjetas de crédito por parte de los estudiantes universitarios

Figure 1. Model proposed to value the likelihood of credit card usage by college students Fuente: elaboración propia basada en las respuestas de los encuestados.

De acuerdo con lo anterior, el procedimiento que se llevó a cabo consistió en la validación de las escalas en las que se midió la consistencia interna del cuestionario y la fiabilidad del instrumento de medida utilizado.

\section{Validez convergente}

La validez de las escalas de medida utilizadas junto con cada uno de los constructos y el instrumento en general, se comprobó a través del método estadístico análisis factorial confirmatorio, dicho análisis representa un conjunto de diversos procedimientos técnicos para el estudio de la relación de interdependencia entre un conjunto de variables con la finalidad de agruparlas en función de la «variabilidad compartida»; descubrir las estructuras subyacentes (factores), cumpliendo la finalidad de resumir y reducir los datos; en otras palabras, esta constituye una técnica estadística muy útil que evalúa la multidimensionalidad de un constructo, ya que permite una exploración empírica, considerando que el objetivo es seleccionar aquellos ítems que correlacionan mayor con el conjunto de ítems que están midiendo el constructo (Pérez \& Medrano, 2010).

Frente a lo anterior, se debe tener en cuenta que la fiabilidad del modelo se valora en dos niveles, por un lado, la fiabilidad de los ítems observables; $y$, por otro lado, se valora la fiabilidad de los constructos (Calvo-Porral, Martínez-Fernández, \& Juanatey-Boga, 2013) y para determinar su impacto sobre el modelo, algunos autores han establecido que una fiabilidad superior a 0.6 es considerada como evidencia de que el modelo es fiable (Bagozzi \& Yi, 1988). Asimismo, la fiabilidad 
de los constructos se refiere al grado con que una variable observable refleja un factor, considerándose aceptable un valor superior a 0.7 (Hair et al., 2007).

Se tiene entonces que la validez convergente evalúa el grado en que la medida de los ítems que recogen un mismo concepto está correlacionada (Calvo-Porral et al., 2013). En el presente estudio no fue necesario eliminar ningún indicador, dado que su carga factorial estandarizada cumple con los criterios de evaluación establecidos por los autores citados previamente, como se observa en la Tabla 1.

Tabla 1. Validez convergente de cargas factoriales estandarizadas Table 1. Convergent validity of standardized factor loadings

\begin{tabular}{|c|c|c|c|}
\hline Constructo & Ítem & $\begin{array}{c}\text { Cargas factoriales } \\
\text { estandarizadas }\end{array}$ & $\begin{array}{c}\text { Promedio de cargas factoriales } \\
\text { estandarizadas }\end{array}$ \\
\hline \multirow{4}{*}{ Beneficios percibidos del crédito } & BP1 & 0.617 & 0.725 \\
\hline & BP2 & 0.739 & \\
\hline & BP3 & 0.792 & \\
\hline & BP4 & 0.753 & \\
\hline \multirow{2}{*}{ Cultura del ahorro } & CA1 & 0.801 & 0.801 \\
\hline & CA2 & 0.801 & \\
\hline \multirow{5}{*}{ Educación financiera } & EF1 & 0.797 & 0.749 \\
\hline & EF2 & 0.806 & \\
\hline & EF3 & 0.700 & \\
\hline & EF4 & 0.781 & \\
\hline & EF5 & 0.662 & \\
\hline \multirow{3}{*}{ Intención de uso de tarjetas de crédito } & IU1 & 0.770 & 0.752 \\
\hline & IU2 & 0.806 & \\
\hline & IU3 & 0.681 & \\
\hline \multirow{3}{*}{$\begin{array}{l}\text { Notificación de costos por parte de la } \\
\text { entidad bancaria }\end{array}$} & NC1 & 0.845 & 0.783 \\
\hline & NC2 & 0.732 & \\
\hline & NC3 & 0.773 & \\
\hline
\end{tabular}

En lo referente a la correlación entre variables, se procedió a calcular la prueba de esfericidad de Barlett y la medida de adecuación de muestreo de Kaiser-Meyer-Olkin -KMO- de adecuación de la muestra y se determinó el nivel de acondicionamiento del modelo para llevar a cabo un análisis factorial. Tanto el valor de KMO como la prueba de esfericidad, detectan la presencia de correlación entre variables y compara las magnitudes de los coeficientes de correlación observados con las magnitudes de los coeficientes de correlación parciales; Kaiser (1974) caracteriza esos valores en una escala que considera a las medidas próximas a 0.90 como maravillosas, a 0.80 como meritorias, a 0.70 como medianas, a 0.60 como mediocres y por debajo a 0.50 como inaceptables (Lévy, Varela, \& Abad, 2006). Se observa en la Tabla 2, que los coeficientes arrojados por el software SPSS para cada uno de los constructos alcanza a cumplir con los criterios mencionados anteriormente, lo cual indica que es factible realizar la técnica de reducción de datos. 
Tabla 2. Validación convergente del KMO y prueba de esfericidad de Bartlett Table 2. Convergent KMO validation and Bartlett's sphericity test

Constructo $\quad$ Valor KMO Valor Bartlett Cumple criterios

\begin{tabular}{llll}
\hline Beneficios percibidos del crédito & 0.683 & 0.000 & Sí \\
\hline Cultura del ahorro & 0.500 & 0.000 & Sí \\
\hline Educación financiera & 0.787 & 0.000 & Sí \\
\hline Intención de uso de tarjetas de crédito & 0.621 & 0.000 & Sí \\
\hline Notificación de costos por parte de la entidad bancaria & 0.633 & 0.000 & \\
\hline
\end{tabular}

Fuente: elaboración propia.

\section{Validez Discriminante}

La validez discriminante es uno de los criterios habituales para evaluar las escalas de medida de constructos latentes en ciencias sociales. En esta fase se afirma que, para que unas medidas sean válidas, las de un mismo constructo deben correlacionar altamente entre ellas, y esta debe ser mayor de la que exista con respecto a las medidas propuestas para otro constructo distinto (Campbell \& Fiske, 1959 citado en Martínez-García \& Martínez-Caro, 2009). El análisis de validez discriminante se ejecutó mediante la comprobación de que el intervalo de confianza en la estimación de la correlación entre cada par de factores no contuviera el valor 1 (Anderson \& Gerbing, 1998). La Tabla 3 evidencia que todos los casos cumplen con dicho criterio y de esta forma se constata que, la validez del instrumento utilizado mide en alto grado aquello que pretendía medir.

Tabla 3. Validez discriminante del modelo de medida

Table 3. Discriminant validity of the measurement model

\begin{tabular}{|c|c|c|c|c|c|}
\hline Constructos & $\begin{array}{l}\text { Beneficios } \\
\text { percibidos del } \\
\text { crédito }\end{array}$ & $\begin{array}{c}\text { Cultura del } \\
\text { ahorro }\end{array}$ & $\begin{array}{l}\text { Educación } \\
\text { financiera }\end{array}$ & $\begin{array}{c}\text { Intención de uso } \\
\text { de tarjetas de } \\
\text { crédito }\end{array}$ & $\begin{array}{c}\text { Notificación de } \\
\text { costos entidad } \\
\text { bancaria }\end{array}$ \\
\hline $\begin{array}{l}\text { Beneficios } \\
\text { percibidos del } \\
\text { crédito }\end{array}$ & 1.000 & & & & \\
\hline $\begin{array}{l}\text { Cultura del } \\
\text { ahorro }\end{array}$ & {$[-0.22 ; 0.262]$} & 1.000 & & & \\
\hline $\begin{array}{l}\text { Educación } \\
\text { financiera }\end{array}$ & {$[0.006 ; 0.214]$} & {$[0.002 ; 0.125]$} & 1.000 & & \\
\hline $\begin{array}{l}\text { Intención de uso } \\
\text { de tarjetas de } \\
\text { crédito }\end{array}$ & [0.248;0.509] & {$[0.002 ; 0.106]$} & {$[0.082 ; 0.313]$} & 1.000 & \\
\hline $\begin{array}{l}\text { Notificación de } \\
\text { costos entidad } \\
\text { bancaria }\end{array}$ & {$[0.099 ; 0.337]$} & {$[0.090 ; 0.249]$} & {$[0.201 ; 0.523]$} & {$[0.089 ; 0.354]$} & 1.000 \\
\hline
\end{tabular}

Fuente: elaboración propia.

Por otro lado, la fiabilidad de la consistencia interna del instrumento se estimó por medio del alfa de Cronbach, en vista de que es una herramienta en la que se asume que los ítems miden a un mismo constructo y están altamente correlacionados (Welch \& Comer, 1988 citado en (Frías, 2013). George 
\& Mallery (2003) citado en Frías (2013) sugieren las recomendaciones siguientes para evaluar los coeficientes de alfa de Cronbach: coeficiente alfa > 0.9 es excelente; coeficiente alfa > 0.8 es bueno; coeficiente alfa $>0.7$ es aceptable; coeficiente alfa $>0.6$ es cuestionable; coeficiente alfa $>0.5$ es pobre y coeficiente alfa $<0.5$ es inaceptable. Sin embargo, en las primeras fases de la investigación un valor de fiabilidad de 0.6 o 0.5 puede ser suficiente. Como se observa en la Tabla 4 , el instrumento de medida parece tener una excelente fiabilidad de la escala de medida, dado que el alfa de Cronbach de los constructos analizados se encuentra entre 0.7 y 0.8 , valores que son bien calificados por los autores mencionados previamente.

Tabla 4. Índice de fiabilidad - Alfa de Cronbach

Table 4. Reliability index - Cronbach's Alpha

\begin{tabular}{lc}
\hline Factor & Alfa de Cronbach \\
\hline Beneficios percibidos del crédito & 0.799 \\
\hline Cultura del ahorro & 0.794 \\
\hline Educación financiera & 0.847 \\
\hline Intención de uso de tarjetas de crédito & 0.792 \\
\hline \multicolumn{2}{c}{ Notificación de costos por parte de la entidad bancaria } \\
\hline \multicolumn{2}{c}{ Fuente: elaboración propia. }
\end{tabular}

\section{Análisis de resultados y contraste de hipótesis}

Para esta etapa de la validación, se procedió a realizar la estimación del modelo estructural propuesto para evaluar la intención de uso de los jóvenes frente a las tarjetas de crédito, en donde se recogen las diversas hipótesis planteadas y se mide su grado de asociación por medio $\mathrm{V}$ de Cramer, los cuales toman valores comprendidos entre -1 y 1 , siendo los próximos a 1 aquellos con una fuerte relación entre las variables contrastadas y los valores próximos a cero las relaciones sin grado de asociación (Abascal \& Grande, 2005). En la Tabla 5 se encuentran los valores obtenidos para los estadísticos de asociación analizados y el modelo utilizado. De acuerdo con la teoría planteada se puede concluir que, los coeficientes de asociación calculados para las relaciones hipotéticas del modelo presentan en su mayoría valores significativos, indicando con esto una correlación adecuada entre las variables evaluadas en el análisis, no obstante, existen relaciones con un grado bajo de asociación, lo que puede reflejar que los constructos contrastados carecen de concordancia para explicar el comportamiento del usuario.

El coeficiente $V$ de Cramer fue extraído del software SPSS y ubicados en una tabla de factores cruzados, para que fuera posible observar el grado de asociación entre las variables que hacían parte de las hipótesis y las que no lo hacían, esto a fin de comprobar el grado de asociación para las relaciones hipotéticas, y al mismo tiempo corroborar que entre los demás constructos no se presenta un nivel de asociación alto. La Tabla 6 evidencia todas las relaciones establecidas entre las variables del modelo propuesto. 
Tabla 5. Contraste de hipótesis. Grado de asociación de factores

Table 5. Hypothesis testing. Degree of association between factors

\begin{tabular}{llc}
\hline Hipótesis & Correlaciones entre variables & V de Cramer \\
\hline H1 & Beneficios percibidos Intención de uso de tarjetas de crédito & 0.359 \\
\hline H2 & Cultura del ahorro Intención de uso de tarjetas de crédito & 0.375 \\
\hline H3 & Educación financiera Intención de uso de tarjetas de crédito & 0.230 \\
\hline H4 & Notificación de costos Intención de uso de tarjetas de crédito & 0.225 \\
\hline H5 & Notificación de costos Cultura del ahorro & 0.299 \\
\hline H6 & Educación financiera Notificación de costos & 0.315
\end{tabular}

Fuente: elaboración propia.

Tabla 6. Coeficiente V de Cramer

Table 6. Cramér's V coefficient

\begin{tabular}{|c|c|c|c|c|c|}
\hline Constructos & $\begin{array}{l}\text { Beneficios } \\
\text { percibidos del } \\
\text { crédito }\end{array}$ & $\begin{array}{l}\text { Cultura } \\
\text { del } \\
\text { ahorro }\end{array}$ & $\begin{array}{l}\text { Educación } \\
\text { financiera }\end{array}$ & $\begin{array}{l}\text { Intención de uso } \\
\text { de tarjetas de } \\
\text { crédito }\end{array}$ & $\begin{array}{l}\text { Notificación de } \\
\text { costos por parte } \\
\text { de la entidad } \\
\text { bancaria }\end{array}$ \\
\hline $\begin{array}{l}\text { Beneficios percibidos del } \\
\text { crédito }\end{array}$ & 1.000 & & & & \\
\hline Cultura del ahorro & 0.230 & 1.000 & & & \\
\hline Educación financiera & 0.164 & 0.285 & 1.000 & & \\
\hline $\begin{array}{l}\text { Intención de uso de tarjetas } \\
\text { de crédito }\end{array}$ & 0.359 & 0.375 & 0.230 & 1.000 & \\
\hline $\begin{array}{l}\text { Notificación de costos por } \\
\text { parte de la entidad } \\
\text { bancaria }\end{array}$ & 0.298 & 0.299 & 0.315 & 0.225 & 1.000 \\
\hline
\end{tabular}

Fuente: elaboración propia.

A continuación, en la Figura 2 se presenta el modelo propuesto con el valor de asociación entre las variables (las líneas indican las hipótesis nulas que se validaron en la investigación).

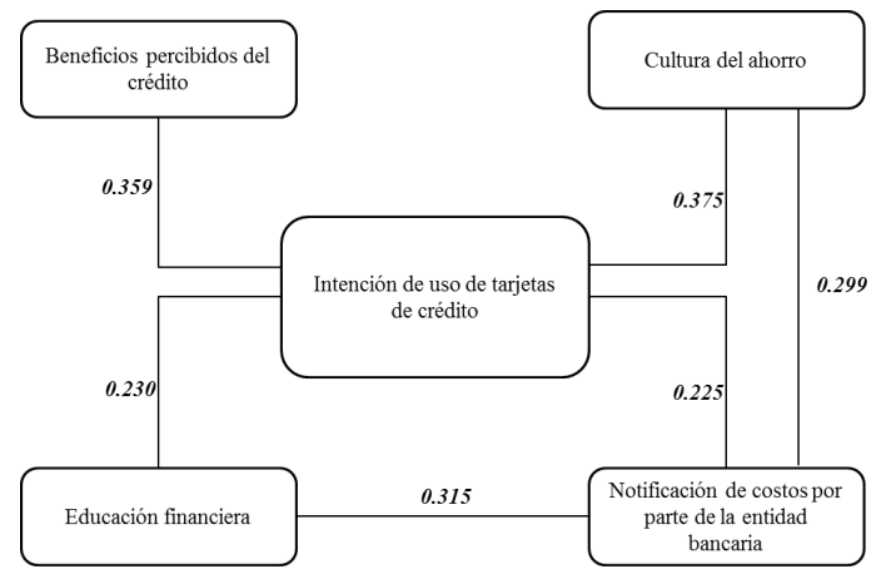

Figura 2. Modelo propuesto, $\mathrm{V}$ de Cramer

Figure 2. Proposed model including Cramér's V

Fuente: elaboración propia. 


\section{DISCUSIÓN}

En el modelo propuesto para esta investigación, se establecen relaciones entre las principales percepciones de los jóvenes universitarios, público objetivo de esta investigación frente la utilización de tarjetas de crédito, para conocer el comportamiento e intención de su uso.

Se encuentra que las relaciones más relevantes a la hora de considerar la intención de participar activamente en el mercado de las tarjetas de crédito están asociadas a la cultura de ahorro y la capacidad de manejar responsablemente el crédito adquirido (0.375) y a la de beneficios percibidos del crédito (0.359). También se observa una fuerte relación entre la educación financiera y planificación del endeudamiento por parte de los usuarios y el sistema de notificación de costos con el que cuenta la entidad bancaria (0.315).

Los resultados enfatizan la relevancia de la cultura del ahorro y los beneficios percibidos del crédito en la intención de uso de las tarjetas de crédito. Según la literatura, es posible percibir que la relevancia de estos constructos coincide con la expectativa temporal y psicológica personal que dan los usuarios sobre lo que consideran su situación económica. Según los resultados, la decisión de gasto parece estar condicionada a si se reduce en el joven universitario la capacidad de pago y de crédito para consumo y a la posibilidad de poder realizar el pago en el plazo pactado.

Las teorías de Duesenberry (1948), citado en Palley (2008), Friedman (1957) y Ganong \& Noel, (2016), parecen configurarse desde enfoques específicos de una enunciación más amplia, en la cual el comportamiento hacia el consumo dependería del seguimiento de un patrón en la toma de decisiones que es construido entre estilos de consumo de usuarios en estratos similares de la sociedad, y de carácter psicológico (Duesenberry, 1948), el cual se establece como un plan equilibrado en el tiempo, y cuyo fundamento es la percepción de seguridad del ingreso y del consumo a futuro. Esta no se ve alterada mientras no se presenten eventos transformadores del esquema lo que coincide con la estrategia de Friedman (Friedman, 1957). Una vez que se presenta un evento, que afecta sustantivamente la posibilidad de ejecutar la planeación financiera prevista, es posible que el usuario con el fin de superar el evento no planeado resuelva los problemas de liquidez con un cambio en su estructura temporal, con soluciones de corto plazo (Ganong \& Noel 2016).

En cuanto al comportamiento de los estudiantes a la hora de usar las tarjetas de crédito, la investigación coincide con Moreno, García \& Gutiérrez (2017), al asegurar que los estudiantes tienen conocimiento y la costumbre de desarrollar presupuestos para planificar sus gastos, pero su nivel de educación financiera es bajo en comparación con el resto de las variables que se evalúan.

Estos resultados sobre la percepción de la población joven son relevantes para las universidades y las entidades bancarias. Para las primeras resultan ser útiles en cuanto a medir el conocimiento impartido en las carreras universitarias y saber cómo reformular con claridad el currículo relacionado con la educación financiera y la cultura de ahorro; y para las entidades, puede ser propicios para el diseño de mejoras en los mecanismos y medios por los cuales notifican los costos y brindan la información asociada a sus productos financieros a los usuarios, como en el caso de las tarjetas de crédito. 


\section{CONCLUSIONES}

La investigación muestra que los estudiantes universitarios son conscientes de la importancia de la educación financiera, a pesar de haber indicado que no habían recibido conocimiento sobre educación financiera por parte de su institución. Lo que deja como recomendación y estrategia institucional, implementar una cátedra sobre educación financiera en los programas de líneas afines, debido a la influencia que esta ejerce en el uso de las tarjetas de crédito y al interés encontrado entre los estudiantes por comprender la utilización de este medio de pago.

Se observa que, las relaciones presentes en el modelo, demuestran que sin lugar a dudas, una de las variables más importantes al momento de fomentar y promocionar el uso de las tarjetas de crédito está estrechamente vinculado con la satisfacción percibida por el cliente, entendiéndose esta desde diversas miradas, que incluyen desde la atención prestada por el asesor corporativo hasta el análisis de la relación entre el conocimiento financiero con el que cuenta el usuario, y la relación costobeneficio que recibe de la entidad al adquirir el crédito.

Un componente fundamental que se relaciona fuertemente con la intención de uso de las tarjetas de crédito es la cultura del ahorro. El estudio evidenció una asociación de 0,375 según el coeficiente de relación estadística $V$ de Cramer. En este sentido, el resultado comprueba una de las teorías encontradas en la literatura en cuanto a que el consumo en el comportamiento de los estudiantes universitarios está relacionado con variables como el crecimiento de la economía personal considerando la intención de uso y con la cultura de ahorro, en cuanto a que gastar más o menos puede estar condicionado a sí se reduce la capacidad de pago y de crédito para consumo, ya que según la investigación las tarjetas de crédito inducen a las personas a realizar compras innecesarias, entendiendo que brindan la posibilidad de anticipar una compra y tener el dinero seguro para pagarla en lapso de tiempo que consideran prudente.

Con la revisión de literatura se pudo identificar los factores influyentes en el uso de las tarjetas de crédito, específicamente reconocer la relación entre la percepción de los estudiantes sobre las ventajas frente al acto de notificar los costos asociados a las tarjetas de crédito por parte de la entidad bancaria, lo cuales según el estudio empírico pueden ser identificados con la influencia del conocimiento sobre la educación financiera, en el sentido en que esta posibilita la toma acertada de decisiones referentes a la planeación de los presupuesto personales, pues según el análisis de los resultados existe una asociación del 0,315 en el coeficiente $V$ de Cramer entre estos dos componentes.

La recolección de la información, a través del contacto con jóvenes estudiantes universitarios, tuvo como resultado información significativa acerca de los factores incidentes en el uso de las tarjetas de crédito, por lo que se resalta la importancia de las percepciones analizadas, ya que se encontró que gran cantidad de estudiantes de carreras relacionadas con el área financiera no han logrado poner en la práctica elementos identificados en los factores, como la educación financiera y la cultura de ahorro, pese a tener la formación. En este sentido, fue importante identificar dichos factores del análisis, ya que con el establecimiento del modelo se contribuye a intentar comprender el comportamiento de los jóvenes universitarios en la intención de uso de las tarjetas de crédito, y constituye la posibilidad de evaluar el potencial para determinar las razones por las cuales dicha población hacer uso de este medio de pago. 
Finalmente, se observa que el estudio sobre el uso de las tarjetas de crédito en los jóvenes universitarios puede ser abordado desde la perspectiva de quien las emite, es decir, analizando las entidades financieras y sus prácticas éticas en el otorgamiento de los altos cupos de este medio de pago, teniendo en cuenta que existen factores que incide en su uso, asociados al comportamiento de consumo, la capacidad adquisitiva y de educación financiera de la población joven que estudia, los cuales son conocidos por dichas entidades, sin embargo no hay investigaciones que estudien el papel que estas juegan en la emisión de las tarjetas de crédito.

\section{REFERENCIAS}

Abascal, A., \& Grande, E. I. (2005). Análisis de encuestas. Madrid; España: ESIC Editorial.

Anderson, J. C., \& Gerbing, D. W. (1998). Structural equation modeling in practice: A review and recommended two-step approach. Psychological Bulletin, 103(3), 411-423. Recuperado de http://psycnet.apa.org/buy/1989-14190-001

Araújo, F. de A. L., \& Souza, M. A. P. de. (2012). Educação Financeira para um Brasil Sustentável Evidências da necessidade de atuação do Banco Central do Brasil em educação financeira para o cumprimento de sua missão. Working Papers Series. Recuperado de https://ideas.repec.org/p/bcb/wpaper/280.html

Bagozzi, R. P., \& Yi, Y. (1988). On the evaluation of structural equation models. Journal of the Academy of Marketing Science, 16(1), 74-94. https://doi.org/10.1007/BF02723327

Banco de la República. (2014). Informe especial de estabilidad financiera- Inclusión financiera. Bogotá. Recuperado http://www.banrep.gov.co/sites/default/files/publicaciones/archivos/iepref_mar_7_2014_0.p df

Calvo-Porral, C., Martínez-Fernández, V. A., \& Juanatey-Boga, S. (2013). Análisis de dos modelos de ecuaciones estructurales alternativos para medir la intención de compra. Investigación Operacional, 34(3), 230-243.

Cano, C. G., Esguerra, M. del P., García, N., Rueda, J. L., \& Velasco, A. M. (2014). Inclusión financiera en Colombia. Banco República. Bogotá. Recuperado de http://www.banrep.gov.co/sites/default/files/eventos/archivos/sem_357.pdf

Del Pino, A., \& Vázquez, E. (2004). El comercio electrónico en España. Una aproximación estadística al fenómeno. Madrid. Recuperado de http://www.revistasice.info/cachepdf/BICE_2798_3145_4EDBF89C5543D00BDCBBA2AB225DBD0B.pdf

Denegri, M., Martínez, G., \& Etchebarne, S. (2007). La comprensión del funcionamiento bancario en adolescentes chilenos: un estudio de psicología económica. Interdisciplinaria, 24(2), 137-159. Recuperado de https://www.redalyc.org/service/redalyc/downloadPdf/180/18024201/1 
Diniz, P., Rogers, P., Barboza, F., \& Mendes-Da-Silva, W. (2016). A Relação da Educação Financeira e do Otimismo no uso de Cartões de Crédito. Revista ESPACIOS, 37(26). Recuperado de http://www.revistaespacios.com/a16v37n26/16372607.html

Duque, E. A., González, J. D., \& Ramírez J. D. (2016). Conocimientos financieros en jóvenes universitarios: caracterización en la Institución Universitaria Esumer. Revista de pedagogía, 37(101). Recuperado de https://www.redalyc.org/html/659/65950543003/

Duesenberry, J. S. (1948). Income-consumption relations and their implications. Lloyd Metzler et al., Income, Employment and Public Policy, New York: WW Norton \& Company, Inc.

Fernández-Corugedo. (2009). Teoría del consumo. México. Recuperado de http://www.cemla.org/PDF/ensayos/pub-en-77.pdf

Frías, D. (2013). Alfa de Cronbach y consistencia interna de los ítems de un instrumento de medida. Universitat de Valencia. Recuperado de http://www.es/friasnav/

Friedman, M. (1957). CHAPTER III. The Income Hypothesis. In Milton Friedman (Ed.). A theory of the consumption function (pp. 20-37). Recuperado de http://www.nber.org/chapters/c4405.pdf

Ganong, P. \& Noel, P. (2016). How Does Unemployment Affect Consumer Spending? Recuperado de https://scholar.harvard.edu/files/ganong/files/ganong_jmp_unemployment_spending.pdf

George, D., \& Mallery, P. (2003). SPSS for Windows Step by Step: Answers to Selected Exercises. A Simple Guide and Reference. https://doi.org/9780335262588

Goldenberg, J. L. (2017). El necesario ajuste de la asignación del riesgo de sobreendeudamiento en la regulación de las tarjetas de crédito: desde un sistema basado en los deberes de información a un modelo de corresponsabilidad. Revista de Derecho, (XLIX), 55-98. Recuperado de https://search.proquest.com/docview/2002988631/fulltextPDF/C4442414DEE54BDAPQ/1?acc ountid $=12268$

Gómez, J. E., Echeverri, J. J., \& Roca, A. M. (2016). Cash usage and trends in credit and debit card payments in Colombia. Recuperado de https://mpra.ub.unimuenchen.de/78005/1/MPRA_paper_78005.pdf

González, E. (2016). Competencia y diferenciación de productos en el mercado mexicano de tarjetas de crédito. Trimestre Económico, 83. https://doi.org/10.20430/ete.v83i332.234

González, L. A. C. (2013). Regulación de las tarjetas de crédito en Costa Rica. Revista de ciencias sociales, (139), 151-160. https://doi.org/10.15517/RCS.V0I139.11361

Hair, J. F., Anderson, R. E., Tatham, R. L., \& Black, W. C. (2007). Análisis Multivariante. 5ta Edición. Prentice Hall International. 
Kaiser, H. F. (1974). An index of factorial simplicity. Psychometrika, 39(1), 31-36. https://doi.org/10.1007/BF02291575

Keynes, J. M. (1943). Teoría general de la ocupación, el interés y el dinero (2 ediciones). Buenos Aires. Recuperado de http://biblio.econ.uba.ar/opac-tmpl/bootstrap/Textocompleto/Teoría general de la ocupación, el interés y el dinero - John Maynard Keynes.pdf

Lévy, J.-P., Varela, J., \& Abad, J. (2006). Modelización con estructuras de covarianzas en ciencias sociales: temas esenciales, avanzados y aportaciones especiales. Modelización con estructuras de covarianzas en ciencias sociales: temas esenciales, avanzados y aportaciones especiales. ISBN 84-9745-136-8. Netbiblo. Recuperado de https://dialnet.unirioja.es/servlet/articulo?codigo=4703924

Limbu, Y. B. (2017). Credit card knowledge, social motivation, and credit card misuse among college students. International Journal of Bank Marketing, 35(5), 842-856. https://doi.org/10.1108/IJBM-04-2016-0045

Limbu, Y. B., Huhmann, B. A., \& Xu, B. (2012). Are college students at greater risk of credit card abuse? Age, gender, materialism and parental influence on consumer response to credit cards. Journal of Financial Services Marketing, 17(2), 148-162. https://doi.org/10.1057/fsm.2012.9

Martínez-García, J. A., \& Martínez-Caro, L. (2009). La validez discriminante como criterio de evaluación de escalas. ¿teoría o estadística? Universitas Psychologica, 8(1), 27-36. Recuperado de https://revistas.javeriana.edu.co/index.php/revPsycho/article/view/224

Mejia M. A., \& Quintero, W. (2017). Determinantes del uso de la banca electrónica en Colombia. Revista Finnova: Investigación e Innovación Financiera y Organizacional, 2(3), 15-26.

Moreno-García, E., García-Santillán, A., \& Gutiérrez-Delgado, L. (2017). Nivel de educación financiera en escenarios de educación superior. Un estudio empírico con estudiantes del área económicoadministrativa. Revista iberoamericana de educación superior, 8(22), 163-183.

Morettini, M. (2002). Universidad Nacional. Principales teorías macroeconómicas sobre el Consumo. Buenos Aires. Recuperado de http://nulan.mdp.edu.ar/1887/1/01486.pdf

Neme-Chaves, S.R \& Forero-Medina, S. (2018) Símbolos, significados y prácticas asociados a las finanzas personales en estudiantes universitarios. Suma de negocios, 9(19) 1-9.

Noreña-Peña, A., Moreno, N. A., Rojas, J. G., \& Malpica, D. M. R. (2012). Aplicabilidad de los criterios de rigor y éticos en la investigación cualitativa. Aquichan, 12(3), 263-274.

Otzen, T., \& Manterola, C. (2017). Técnicas de Muestreo sobre una población a Estudio. International Journal of Morphology, 35(1), 227-232.

Pacheco-Bernal, D. J., Segovia-Baquero, S. D., \& Yaruro-Jaime, A. M. (2017). Vulnerabilidades financieras de los hogares en Colombia. Borradores de Economía, 1026. Recuperado de 
http://www.banrep.gov.co/sites/default/files/publicaciones/archivos/borradores_de_economi a_1026.pdf

Palley, T. I. (2008). The Relative Income Theory of Consumption: A Synthetic Keynes-DuesenberryFriedman Model (170). Massachusetts. Disponible en: www.peri.umass.edu

Pérez-Gil, J. A., Chacón, S., \& Moreno, R. (2000). Validez de constructo: el uso de análisis factorial exploratorio-confirmatorio para obtener evidencias de validez. Psicothema, 12(SUPPL. 2), 442446. https://doi.org/10.1177/0013164412473825

Pérez, E. R., \& Medrano, L. (2010). Análisis factorial exploratorio: Bases conceptuales y metodológicas. Revista Argentina de Ciencias Del Comportamiento, 2(1889), 58-66. https://doi.org/10.2307/2183556

Raccanello, K., \& Herrera Guzmán, E. (2014). Educación e inclusión financiera. Revista Latinoamericana de Estudios Educativos (México), 44(2).

Reina, M., Zuluaga, S., \& Rozo, M. (2006). El dinero y la política monetaria. Recuperado de https://s3.amazonaws.com/academia.edu.documents/39253302/guia2_el_dinero_y_la_politic a_monetaria.pdf?AWSAccessKeyId=AKIAIWOWYYGZ2Y53UL3A\&Expires=1554224577\&Signatu re=7MaUrF3f9RPADEdTInfqakwa4xY\%3D\&response-contentdisposition=inline\%3B\%20filename\%3DGuia2_el_dinero_y_la_politica_monetaria.pdf

Rivera B. E., \& Bernal, D. (2018). La importancia de la educación financiera en la toma de decisiones de endeudamiento. Estudio de una sucursal de «Mi Banco», en México. Revista Perspectivas, (41), 117-144.

Rivero, G. E., Rosado, Y. L., \& Pérez, A. E. (2017). El uso de tarjetas de crédito en las finanzas personales. Caso trabajadores administrativos de una institución de educación. $V$ Congreso Virtual Internacional sobre Transformación e innovación en las organizaciones. Llevado a cabo en México.

Singh, S., Rylander, D. H., \& Mims, T. C. (2018). Understanding credit card payment behavior among college students. Journal of Financial Services Marketing, 23(1), 38-49. https://doi.org/10.1057/s41264-018-0042-0

Solano, M. D. C., Vega, L. V., \& Cárdenas, L. F. (2015). Determinantes del uso de tarjetas de crédito en México. Reflexiones sobre el papel de la cultura financiera. Revista Raites, 1(1), 61-76.

Wang, A. (2011). Effects of Gender, Ethnicity, and Work Experience on College Students' Credit Card Debt: Implications for Wealth Advisors. The Journal of Wealth Management, 14(2), 85-100. https://doi.org/10.3905/jwm.2011.14.2.085

Xiao, J. J., \& O'Neill, B. (2016). Consumer financial education and financial capability. International Journal of Consumer Studies, 40(6), 712-721. https://doi.org/10.1111/ijcs.12285 